\title{
Philosophiques
}

\section{Michel Morin, Mort et résurrection de la loi morale, Montréal, Collection Constantes, Éditions Hurtubise HMH, 1997, 170 p.}

\section{Marc Bélisle}

Volume 28, numéro 1, printemps 2001

La nature des normes

URI : https://id.erudit.org/iderudit/004900ar

DOI : https://doi.org/10.7202/004900ar

Aller au sommaire du numéro

Éditeur(s)

Société de philosophie du Québec

ISSN

0316-2923 (imprimé)

1492-1391 (numérique)

Découvrir la revue

Citer ce compte rendu

Bélisle, M. (2001). Compte rendu de [Michel Morin, Mort et résurrection de la loi morale, Montréal, Collection Constantes, Éditions Hurtubise HMH, 1997, 170 p.] Philosophiques, 28(1), 223-226. https://doi.org/10.7202/004900ar d'utilisation que vous pouvez consulter en ligne.

https://apropos.erudit.org/fr/usagers/politique-dutilisation/ 
Michel Morin, Mort et résurrection de la loi morale, Montréal, Collection Constantes, Éditions Hurtubise $\mathrm{HMH}, 1997,170 \mathrm{p}$.

Les ouvrages de philosophes professionnels (ou disons simplement universitaires) étant devenus très techniques ou pour le moins scolaires, une tentative de faire une oeuvre philosophique qui cherche à penser le quotidien en dehors des cadres académiques apparaîtra aux dilettantes et aux non-initiés comme la bienvenue. C'est à l'intérieur de cet espace que s'est constituée l'œuvre de Michel Morin (déjà huit ouvrages parus) et son dernier livre, Mort et résurrection de la loi morale, persiste et persévère dans cette voie.

De quoi y est-il question ? Le titre l'annonce : de la fin de la morale comme " corps de doctrines ou de lois formulées a priori et à l'intention de tous »(11) et de la possibilité de faire naître, du sein de l'individu, une autre morale, une nouvelle exigence éthique. Considérant la place de la réflexion éthique à l'intérieur du discours philosophique en général, on évaluera l'ambition du projet qui veut recommencer, reprendre à neuf cette question ; cependant son point de départ inquiète : " cherchant encore, persévérant, peut-être parviendra-t-on à trouver quelque chose de plus impératif, mais alors surgi du coeur de soi-même, une sorte de loi, qui serait issue d'une passion, d'une exigence, d'une nécessité intérieure assez forte pour nous tenir, nous retenir (d'aller dans tous les sens) et nous contraindre à suivre résolument telle direction, sans peut-être savoir pourquoi ni où elle pourrait bien nous mener, mais avec cette conviction, osons le mot, cette foi que l'on accorde qu'à ce qui est issu du plus profond de soi » (12). Si l'auteur affirme reprendre à son compte le constat de Nietzsche selon lequel les hommes ne vivent plus en fonction de projets universalistes et donc métaphysiques, il ne semble pas s'appuyer sur les mêmes prémisses : la fin des idéaux, la désagrégation de l'unité, la fausseté de la vérité. Il faut se demander si on ne sème pas la confusion quand on voit ici la passion, l'intériorité, le " plus profond 


\section{Philosophiques / Printemps 2001}

de soi ", être compris sous la catégorie de la loi, c'est-à-dire de l'exigence, de l'unité d'orientation et de l'impérativité. Il s'agirait de retrouver le sens de la loi, en nous (et non la loi comme telle), à partir du chaos qui nous habite; et dans cette perspective les distinctions traditionnelles ne seraient plus pertinentes. L'auteur se propose de penser devant nous ces retrouvailles en s'inspirant de la lectures d'auteurs qui lui tombent sous la main.

Ces auteurs - Nietzsche, Héraclite, Eschyle et Sophocle — ont bien sûr été choisis... en fonction de leur capacité de féconder l'auteur. Si l'on pouvait s'attendre, au seul regard de la table des matières, à se voir entre autre proposée une interprétation du Zarathoustra, on est vite déçu. La reprise du « Dieu est mort » et de la remise en question des idéaux traditionnels ne sert qu'à mettre en question le rapport non personnel, non intérieur, bref inauthentique que le monde actuel entretient face aux valeurs. Le rapport authentique ne serait possible que sur le fond d'une connaissance de soi non rationnelle, mais d'abord comme chaos et inquiétude. C'est pourquoi c'est son «drame intérieur » qui sera de Nietzsche retenu et exposé. Le problème de cette reprise des thèses nietzschéennes c'est qu'elle laisse croire qu'il y a déjà eu quelque chose - " une assemblée de croyants ", une communauté avec des valeurs absolues — qui n'est plus possible aujourd'hui et à laquelle on doit apprendre à dire "non » pour parvenir à "cela au fond de soi, qui est vraiment soi » (27). On pose comme nécessaire une traversée du "désert intérieur ", une ascèse, qui devient le sceau du rapport authentique à soi et à la loi. Ce faisant on se prévient contre toute discussion ou remise en question puisqu'il s'agit d'exprimer la différence irréductible de notre individualité, garantie par notre souffrance.

Si l'on voulait seulement affirmer que l'individu est renvoyé à lui-même en face du monde, on n'aurait rien dit de bien nouveau : toute la tradition a toujours répété qu'une pensée ou un jugement devait être réeffectué, actualisé ou repensé par celui auquel il est adressé pour prendre tout son sens et devenir efficace. Cependant l'individu dont il est ici question n'est pas un interlocuteur dans l'espace de la parole, il est cette singularité irréductible, inscrite dans la chair et le corps comme "nouvelle source de valeurs » (?!). Dès lors que nous reste-t-il à nous dire entre nous que la différence de nos désirs (toujours déçus) et la douleur de cette différence en elle-même incommunicable ? Faire la critique de ce livre serait alors porter atteinte à l'intégrité souffrante de celui qui l'a écrit, l'expression d'un vieux moraliste, plein de ressentiment.

Le chapitre inspiré par Héraclite est de ce point de vue fort significatif. La méditation personnelle des fragments d'Héraclite nous permettrait de remettre en question notre rapport à la représentation de ce qui pourrait satisfaire notre désir. Par-delà l'anachronisme qu'implique cette critique de la représentation supposée chez un présocratique, on admettra (avec Freud, qui n'est pourtant pas évoqué) que notre désir premier, originaire, est inconscient et que sa représentation est toujours trompeuse. On admettra peut-être aussi, bien que de façon plus problématique, que ce qui advient à travers lui, à travers son échec, est réel, la vraie réalité. Mais affirmer que cette réalité, c'est la Nature, parce que " Nature aime à se cacher », est pour le moins déconcertant, d'autant que cette Nature est évoquée pour inviter à la reconnaissance d'un désir assez loin de la nature : " puis-je consentir à ce que, prétendant désirer des personnes du même sexe que moi, je ne me sois jusqu'à maintenant intéressé qu'à des personnes ouvertes sur l'autre sexe » (80). À travers la question du désir, ce sont l'ambivalence et l'ambiguïté assumées qui apparaissent comme les moments du " plus grand éveil possible de la conscience », permettant même à l'auteur d'exprimer 
de manière plus explicite la valeur et la lucidité qu'il s'accorde, de soutenir qu'à travers son "drôle de désir ", il vit " ouvertement ce que les autres dissimulent " (81), à savoir un rapport authentique à l'altérité que les « endormis » vivent sous le mode de l'identité rêvée. Bien que le texte soit admirablement bien écrit et parsemé d'envolées poétiques - il suffit de lire la description de la conscience, déçue dans son attente et ravie en même temps de ce qui advient au plus près de soi, dans l'intime, qui brûle d'un feux doux dans l'oubli de soi (68-71), pour s'en convaincre - le philosophe ne peut en tirer grand chose. Qu'en plus on se serve de cette " lecture du désir » pour condamner, du point de vue politique, le projet souverainiste sous prétexte qu'il ne chercherait que la satisfaction de son désir à travers la représentation de celui-ci, sans être capable d'accueillir l'autre de son désir de l'intérieur même de celui-ci, relève pour le moins d'une confusion catégorielle.

Puis voilà que les grands tragiques sont convoqués. L'Antigone de Sophocle en premier lieu, pour lui faire exprimer ce qu'il en est de l'écoute de la voix intérieure et de l'assomption de son destin, de sa nature, contre la force coercitive et communautaire de son beau-père Créon. Ici la parole est clairement comprise comme simple expression de la douleur, de la souffrance et de la solitude. La communication est impossible, seulement le reconnaissance : la reconnaissance de ce que je te fais souffrir du fait d'affirmer ma différence. Et ainsi se poursuit un enfoncement dans la « parole des dieux ", introduite déjà par la lecture d'Héraclite, qui frôle la mystique de la Terre et du sang. Puis enfin, et c'est là la seconde partie du livre (qui ne compte que pour un tiers), on propose de comprendre l'Orestie d'Eschyle comme une critique de l'intégrisme et de la «maternisation de la loi ». Agamemnon représente la Loi du Père qui s'élève et s'affirme au-dessus de la tribu et de liens affectifs pour faire sentir l'exigence de l'aventure et de l'universel ; mais son trône est usurpé pendant son exil conquérant qu'est la guerre de Troie, et sa femme le tue à son retour. C'est son fils Oreste qui devra accomplir sa vengeance pour rétablir la nécessité de la Loi du père contre la loi de la mère, tout en assumant la mort de ce père : belle métaphore pour reprendre le thème dès le départ annoncé : la loi ancienne est morte et on ne peut pas la faire revivre ni y retourner, sinon la ressusciter comme exigence au fond de soi et de son être intérieur. Le règne de la mère, c'est l'enfoncement dans la sensibilité et la «pourriture » dont il faut se dégager parce qu'il ne remplace rien et n'est que dénégation.

Le problème le plus important que cette lecture suscite, c'est qu'elle comprend l'accès à la raison comme une exigence, ce qu'elle a toujours été, en même temps qu'elle dénie les effets de cette découverte en soi de la raison : on affirme du même souffle la nécessité d'un dépassement de la sensibilité (dans laquelle toute la première partie nous avait confiné) et l'irréductibilité de sa différence personnelle. Cette notion de différence n'étant jamais thématisée philosophiquement ou ontologiquement s'agirait-il de la différence entre l'être et le langage ? - elle ne peut servir d'appui à aucun jugement. Si la nécessité de la loi est reconnue, on ne veut l'affirmer que comme loi qui ne vaut que pour soi, qui n'a de valeur que dans un rapport authentique et personnel à elle et qui, par là, neutralise sa fonction même de loi. On pourra accuser l'Université de mal accueillir de telles œuvres ; mais il faudrait d'abord se demander comment on peut accueillir une oeuvre qui exclue la possibilité d'un dialogue. Si la découverte en soi de la raison est exigence et contrainte, c'est qu'elle oblige à faire place à l'autre, aux autres et au monde extérieur et non à fantasmer en soi un autre que soi. On constate plutôt ici qu'une structure paranoïaque s'est mise en place : tout ce qui vient de l'extérieur est mauvais et mortel, alors que ce qui vient de soi est bon ; 


\section{6 . Philosophiques / Printemps 2001}

il faudra cependant se méfier et distinguer en soi le soi qui vient de l'extérieur (le mauvais) du soi réel qui m'apparaît de prime abord comme un autre (celui-là est le bon).

Les dernières pages (160-161) essaient de redonner un "sens métaphysique à la souffrance » d'être dans cette différence solitaire, séparé des autres hommes, à faire valoir cette séparation comme un mal sans remède parce que ni psychologique, ni physiologique. N'y a-t-il pas là reconnaissance implicite de la folie que peut constituer une tentative personnelle et singulière de philosopher ?

MARC BÉLISLE

Cégep Édouard-Montpetit 